\title{
Clasificación de los mejores programas de pregrado de Administración en Colombia según criterios de investigación: periodo 2016-2017
}

\section{Ranking of the best undergraduate programs in Administration in Colombia based on the research criteria from period 2016-2017}

\section{Elkin Fabriany Pineda-Henao' José Londoño-Cardozo ${ }^{2}$}

Recibido: marzo 26 de 2018 Aceptado: junio 28 de 2018

\section{Resumen}

Este documento presenta una propuesta para clasificar las facultades de administración colombianas con programas de pregrado a partir de indicadores de generación de conocimiento. El trabajo se desarrolló con parámetros cuantitativos en el que se realizaba un proceso de análisis comparativo e interpretativo de la información contenida en rankings académicos especializados del año 2017, así como de los datos obtenidos de los grupos de investigación y de los posgrados de las mejores universidades que se encuentran consignados en sus sitios web y en la plataforma GrupLac de Colciencias. La clasificación final contiene diecinueve programas y entrega un panorama del estado de algunos de los mejores programas de pregrado en Administración de Colombia. Se concluye que es necesario mejorar la razón programas posgraduales versus calidad y cantidad de grupos de investigación, lo cual permitiría a las facultades de Administración con peores posicionamientos mejorar sus indicadores de calidad de sus programas de pregrado.

Palabras clave: producción científica, investigación en administración, escuelas de negocios, educación superior, conocimiento administrativo.

\begin{abstract}
This document presents a proposal to classify the Colombian administration faculties with undergraduate programs based on indicators of knowledge generation. This study was developed under quantitative parameters in which a process of comparative and interpretative analysis of the information contained in specialized academic rankings of the year 2017 was carried out, as well as of the data obtained from the research groups and postgraduate programs of the best universities that are consigned in their websites and in the GrupLac platform of Colciencias. The final classification contains nineteen programs and provides an overview of the status of some of the best undergraduate programs in Administration in Colombia. It is concluded that it is necessary to improve the ratio of postgraduate programs versus quality and quantity of research groups, which would allow the Faculty of Administration with the worst positions to improve their quality indicators of their undergraduate programs.
\end{abstract}

Keywords: scientific production, research in administration, business schools, higher education, administrative knowledge.

1 Licenciado en Filosofía y Administrador de Empresas, Magíster en Administración, Universidad del Valle, Palmira, Colombia. E-mail: elkin.pineda@correounivalle.edu.co

2 Tecnólogo Industrial, Estudiante Administración de Empresas, Universidad Nacional de Colombia, Palmira, Colombia. E-mail: jodlondonoca@unal.edu.co 


\section{Introducción}

A pesar del avance en los estudios sobre la investigación en Administración en Colombia, existe aún un vacío en temas particulares (Calderón-Hernández, Gutiérrez-Vargas \& Castaño-Duque, 2017). La mayoría de estos estudios se concentra en las escuelas de administración como un todo, y en la investigación realizada en programas de posgrado, por lo que un tema en que aún se perciben carencias se refiere al lugar y la importancia de la formación investigativa en estudiantes de pregrado, así como la manera en que la investigación influye en los procesos formativos de los estudiantes de Administración.

Algunos estudios en Latinoamérica sugieren que la calidad y el éxito de egresados de escuelas en Administración, está vinculado a la calidad de la formación investigativa de la planta docente y a la producción intelectual de los grupos de investigación (Gantman, 2013). Si se sigue dicha hipótesis, resulta loable rastrear la influencia que podría tener en la formación de estudiantes de pregrado en Administración, la cantidad y la calidad de los grupos de investigación y de posgrados de una misma escuela.

El presente artículo hace parte de los resultados de tres proyectos de investigación financiados por la Universidad Nacional de Colombia, UNAL, en las convocatorias a) Programa nacional de iniciación en investigación, creación e innovación de la UNAL 2013-2015, y b) Convocatoria nacional de proyectos para el fortalecimiento de la investigación, creación e innovación de la UNAL 2016-2018, liderados por el Grupo de Estudios Neoinstitucionales - GEN. Estos estudios indagan la problemática de la formación en administración y su relación con la investigación, con el fin de hallar directrices de mejora para el programa de Administración de Empresas de la UNAL, Sede Palmira. Los dos primeros se titulan i) Análisis de las competencias profesionales en la formación de estudiantes de pregrado en la carrera de Administración de empresas en Colombia: perspectivas de algunos expertos; y ii) Perfiles de formación profesional en Administración: identificación, caracterización y contrastación en algunas universidades colombianas, desarrollados entre 2015 y 2016. El más reciente se titula iii) Estudio comparativo nacional de las tendencias temáticas de investigación en la administración y su influencia en la formación universitaria, desarrollado entre 2017 y 2018.

El problema aquí abordado se refiere a la clasificación de las escuelas de Administración, que contienen programas de pregrado, en términos de indicadores de investigación. Al respecto, se propone un análisis comparativo de algunos rankings y de datos obtenidos de los grupos de investigación y los posgrados de las mejores universidades colombianas, a partir de sus sitios web y de la plataforma ScienTi GrupLac de COLCIENCIAS. Estos datos fueron ponderados para crear un listado de mejores universidades con programas de Administración, de acuerdo a indicadores de investigación, para el año 2017.

Los resultados muestran tres listados de diecinueve universidades, de acuerdo a los indicadores mencionados. El primero de ellos ordena estas universidades de acuerdo a una ponderación realizada de la cantidad y calidad de los grupos de investigación, con datos de la Convocatoria 737 de Colciencias (2015). El segundo listado las ordena de acuerdo a la ponderación resultante de la cantidad y tipo de programas de posgrado, con datos de las páginas web de las universidades y vigentes hasta el año 2017. Finalmente, se propone un listado unificado que suma las ponderaciones realizadas en los dos listados anteriores, ordenando de mayor a menor a dichas universidades.

\section{Marco teórico y métodos}

\subsection{Referentes conceptuales}

Investigación y calidad formativa en escuelas de Administración

Una de las premisas latentes en el paradigma actual de la calidad en la formación universitaria, es que ésta última debe tener una importante influencia del desarrollo investigativo de las dis- 
ciplinas. Al respecto, se ha argumentado que organismos nacionales como el Consejo Nacional de Acreditación, CNA, y el Ministerio de Educación Nacional de Colombia, MEN, asumen que la investigación es un elemento crucial para la calidad de los programas de pregrado (Aldana-de Becerra, 2012).

EI MEN considera que, debido a lo anterior, los programas: "(...) deben tener actividades de investigación que permitan desarrollar una actitud crítica y una capacidad creativa para encontrar alternativas para el avance de la ciencia, la tecnología, las artes y las humanidades en el país" (MEN, 2010). Esto hace parte del sustento teórico por el cual los procesos de acreditación institucional, han incluido criterios exigentes sobre el aspecto investigativo para garantizar la formación de calidad. En palabras de Aldana de Becerra:

(...) los procesos de acreditación y reconocimiento de alta calidad de las Instituciones de Educación Superior (IES) están relacionados con investigación. La normatividad colombiana plantea que las universidades de educación general, de docencia o de profesionalización, si aspiran a ser universidades, deben tener algún grado de investigación, reflejada en número de publicaciones científicas, en el impacto de éstas y en la cantidad de institutos o centros de investigación reconocidos; a la universidad se le declara ente social por excelencia para construir y reconstruir conocimiento y para formar profesionales comprometidos con los problemas sociales. (Aldana-de Becerra, 2012, p. 369.)

El compromiso de la investigación con la formación universitaria no sólo obedece al cumplimiento de estándares para la consecución de acreditaciones de calidad. En el fondo, se trata de una cuestión mucho más importante que señala la esencia misma de la existencia de las universidades: la misión institucionalizada de estas ante la sociedad remite a que la generación de conocimientos tenga una incidencia en la solución de los problemas sociales y humanos, que redundan en el desarrollo cultural y económico (Aldana-de Becerra, 2012).

El anterior referente, contextualizado a las escuelas de Administración, se encuentra en sincronía con estudios latinoamericanos que sugieren una correlación entre el nivel de investigación y la calidad y el éxito de sus egresados. Según la investigación de Gantman (2013) con las mejores escuelas latinoamericanas de negocios, la producción intelectual de los grupos de investigación de éstas junto con la formación doctoral de sus docentes, posee una relación determinante con el triunfo de sus egresados. Específicamente, este autor sostiene que:

(...) Los resultados indican que el nivel de producción académica de las escuelas, el cual se obtuvo del número de artículos en publicaciones indexadas en el Social Science Citation Index, está asociado en forma positiva y estadísticamente significativa con el éxito de sus egresados. Además, la calidad de la planta docente también tiene un efecto positivo y significativo sobre dicha variable, aunque este último hallazgo no es robusto, de acuerdo con las distintas especificaciones de los modelos de regresión. (Gantman, 2013, p. 279.)

La correlación estadística planteada por Gantman (2013), resulta interesante y da apertura a otros planteamientos que busquen explicarla. Por ejemplo, es posible que la conexión que da paso al éxito de los egresados, se manifieste por la influencia que tenga la formación de alta calidad de los docentes y la producción intelectual de los grupos, con las actividades de formación dadas a los estudiantes, previo a su egreso. Así, dicha influencia podría darse puntualmente en los contenidos y las materias, o en la participación de los estudiantes en semilleros de investigación; lo que permitiría a los estudiantes de Administración desarrollar ciertas habilidades del pensamiento, como el razonamiento crítico y la creatividad, que complementarían la hegemónica formación práctica. 
Algunas consideraciones sobre la investigación en Administración en Colombia

Según Calderón-Hernández et al. (2017), pese a que la investigación en Administración en el país tuvo fuertes retos hace varios años, en la actualidad goza de un desarrollo importante que, no obstante, aún está sujeto de mejora. En un inicio, trabajos como los de Dávila $(1982 ; 1986 ; 1988)$ y Ogliastri (1982), mostraban un escenario con grandes dificultades para la producción intelectual en Administración, que dio paso a la preocupación por el desarrollo de dicho campo de estudios (Calderón-Hernández et al., 2017).

Igualmente, Calderón-Hernández et al. (2017), sostienen que otros estudios hicieron seguimiento a esta problemática y develaron grandes dificultades relativas a la consolidación de los grupos, sus líneas de investigación y la producción intelectual (Malaver-Rodríguez, 1999). A pesar de esto, Calderón Hernández et al. (2017) aluden que años más tarde se advierte un avance importante de la investigación en Administración en Colombia entre los años 2000 y 2006 (Malaver-Rodríguez, 2006) el cual luego parece ser progresivo desde 1965 hasta 2015 (Malaver-Rodríguez \& López-Gallego, 2016).

Además, Calderón-Hernández et al. (2017), resaltan que dicho avance en la actualidad se ve reflejado por un aumento en los grupos de investigación, su consolidación y su producción intelectual (Calderón -Hernández et al., 2014). Sin embargo, aún existen diversas temáticas por resolver, entre esas las asociadas con el estado de las escuelas de Administración (Calderón-Hernández et al., 2017) y los vínculos que ello puede tener con la formación investigativa y la calidad en pregrado. Por otro lado, en cuanto a la formación posgradual en Administración en Colombia, Salcedo Serna, Londoño Cardozo \& Hernández Madroñero (2017) sostienen que han aumentado en Colombia, pese a que aún falta un mayor avance en la formación doctoral en comparación con otros países.
Finalmente, se debe resaltar que la preocupación por el avance de la investigación en Administración en Colombia ha ido acompañada de reflexiones de corte filosófico. Algunas de estas discusiones se han enfocado en cuestionar o fundamentar el carácter científico de la disciplina administrativa (Cornella-Solans, 1998; Flórez, 2011; Marín-Idárraga, 2012; Pineda-Henao, 2012; 2015; 2017; 2018a; Pineda-Henao \& Tello-Castrillón, 2018). Otras, han esgrimido posturas sobre las distinciones básicas entre ciencia y tecnología (Durango-Yepes, 2008; Pineda-Henao, 2012; 2015; 2017; 2018a; Pineda-Henao \& Tello-Castrillón, 2018) que dan paso a la distinción y relación entre el pensamiento y la acción administrativa, mientras que otras reflexiones han manifestado diversos problemas discursivos, teóricos y terminológicos (Cruz-Kronfly, 1982; Marín-Idárraga, 2012). También han surgido planteamientos sobre la diversidad de objetos de estudio en este campo (Marín-Idárraga, 2012) y sobre los fundamentos de la realidad de dichos objetos (Carvajal, 2010; Pineda-Henao, 2013; 2014a; 2014b).

\subsection{Metodología}

La presente investigación se llevó a cabo bajo parámetros fundamentalmente cuantitativos, con su respectivo análisis interpretativo devenido de la revisión documental. Se realizó un proceso de análisis comparativo e interpretación de la información contenida en los rankings académicos especializados y datos obtenidos entre los años 2016 y 2017, de los grupos de investigación y los posgrados de las mejores universidades, a partir de sus sitios web y de la plataforma GrupLAC, de COLCIENCIAS. Se decidió que la información a obtener para realizar esta investigación cumpliera los siguientes criterios: i) grupos de investigación, y ii) programas de posgrado, pues, en consonancia con el referente conceptual, eran los datos públicos más próximos a la producción intelectual y la formación de alta calidad de la planta docente.

Para cumplir con el objetivo, se incluyeron las mejores universidades del país, seleccionadas bajo 
criterios de: calidad, visibilidad e investigación, por dos rankings especializados en el tema, que cuentan con gran reconocimiento entre la comunidad académica. A continuación se describe este proceso.

En un primer momento se selecciona el ranking publicado por Sapiens Research (2017a) para determinar las universidades mejor calificadas. Se tomaron las universidades equivalentes a los cuartiles 1,2 y 3 . A este grupo se adicionó la Universidad Nacional de Colombia, Sede Palmira, del cuarto cuartil, por ser la ejecutora del proyecto con la cual se realizaría la comparación. Luego se identificaron los programas de administración del país con mejores indicadores, según los criterios antes mencionados.

Se consultó el ranking de Sapiens Research (2017b) para seleccionar los programas de administración según criterios de calidad. Este ranking le asigna a cada programa una calificación en cuatro categorías: a) AAA, b) AA, c) A y sin calificación. Se seleccionaron los programas de Administración con calificación AAA, los cuales son aquellos que bajo los criterios del ranking están posicionados en los más altos estándares de los criterios seleccionados. Con esta información se seleccionaron las universidades del estudio.

Una vez obtenidas estas dos listas, se realizó un cruce de información entre ambas para verificar que universidades de la lista $A$ tenían un programa acreditado en la lista B. Este proceso permitió seleccionar las diecinueve universidades que coincidieron en ambos rankings.

La búsqueda de información para las universidades seleccionadas, se adelantó en dos sitios. En primer lugar se ingresó a las páginas web institucionales de cada una y se tomó la información de: a) los programas de posgrado de Administración ofertados hasta 2017 por la facultad a la que pertenece; b) la información relativa a los grupos de investigación en Administración que declara cada programa hasta 2017. En el caso del nume- ral a) solo se tuvieron en cuenta los posgrados que generan nuevo conocimiento a través de la investigación; es decir, las maestrías y los doctorados (Hernández-Martínez, Saavedra-Mayorga \& Sanabria-Rangel, 2006). Se tuvo en cuenta que una escuela o facultad de Administración puede ofrecer distintos programas en disciplinas afines (Londoño-Cardozo, 2018) que pertenecieran a la Administración o a sus áreas funcionales.

En el numeral b), se tomó la información de todos los grupos de investigación adscritos al programa de Administración. Esta información se contrastó con la registrada en los GrupLAC. Solo se tuvieron en cuenta los grupos de investigación con clasificación según la convocatoria 737 de Colciencias (2015) que aún estaban vigentes para el año 2017. Un panorama completo sobre los grupos de investigación de las universidades en estudio, se encuentra en el trabajo de Hernández-Madroñero, Londoño Cardozo y Pineda-Henao (2018).

En la creación del ranking de las universidades según sus grupos de investigación, se utilizó la metodología empleada por Calderón-Hernández et al. (2017). Se ordenaron los grupos de cada universidad según las categorías de Colciencias: A1, A, B, $C$ y D. A cada categoría se aplicó una ponderación: "A $1 * 9.0+A^{*} 7.0+B^{*} 4.0+C^{*} 2.0+D^{*} 0.1$ " (Calderón-Hernández et al., 2017, p. 49). Al total de grupos en cada categoría se multiplicó por el factor asignado y se sumaron los totales.

En el caso de los programas de posgrado, la información fue depositada en una tabla, en la que se discriminó la cantidad y el tipo de programa ofertado por cada facultad. Al igual que con los grupos de investigación, se les aplicó una ponderación para determinar una clasificación. Se asignó un puntaje según el tipo de programa posgradual. A los doctorados se les dio 10 puntos y a las maestrías 5 puntos. La multiplicación de los puntos por el número de programas ofertados por tipo se sumó para cada universidad, con lo que se crea un ranking. 
Estos dos consolidados, el de las universidades según grupos de investigación y según oferta de posgrados, se llevaron a una matriz en Excel. Una vez allí, a cada criterio, i) grupos de investigación y ii) programas de posgrado, se le dio una ponderación del 50\%. Esta cantidad porcentual fue seleccionada al no contar con una teoría o investigación que permitiese valorar la mayor o menor importancia de cada uno de los criterios sobre el otro y para evitar parcialidades. En cada universidad se multiplicó sus dos indicadores por la ponderación. El resultado de esto se sumó y se organizó de mayor a menor, donde el mayor es la universidad número uno, según estos criterios, y el menor la última universidad. De esta forma se generó el ranking que se presenta en los resultados.

\section{Resultados y discusión}

A partir de la metodología antes descrita, se seleccionaron las diecinueve universidades que se presentan en la Tabla 1. Éstas comprenden una muestra heterogénea de diferentes regiones del país. Entre ellas se encuentran universidades de Bogotá y su área de influencia, Medellín, la costa caribe y el pacífico, con Cali y Popayán.

\begin{tabular}{lll}
\hline $\begin{array}{l}\text { posición en } \\
\text { el Ranking } \\
\text { nacional }\end{array}$ & Universidades que abarca el estudio & \\
\cline { 2 - 3 } & Universidad & Ciudad \\
\hline 2 & Universidad Nacional de Colombia & Bogotá \\
\hline 3 & Universidad de Antioquia & Medellín \\
\hline 4 & Universidad de Los Andes & Bogotá \\
\hline 5 & Universidad del Valle & Cali \\
\hline 8 & Pontificia Universidad Javeriana & Bogotá \\
\hline 9 & Universidad del Norte & Barranquilla \\
\hline 11 & Universidad Pedagógica y Tecnológica de Colombia & Tunja \\
\hline 14 & Universidad Pontificia Bolivariana & Medellín \\
\hline 15 & Universidad del Cauca & Popayán \\
\hline 16 & Universidad de Cartagena & Cartagena \\
\hline 17 & Universidad de Medellín & Medellín \\
\hline 19 & Universidad Externado de Colombia & Bogotá \\
\hline 20 & Universidad de la Sabana & Chía \\
\hline 23 & Universidad del Rosario & Bogotá \\
\hline 26 & Universidad Militar Nueva Granada & Bogotá \\
\hline 29 & Universidad Santo Tomás & Bogotá \\
\hline 32 & Universidad de la Salle & Bogotá \\
\hline 40 & Universidad EAN & Bogotá \\
\hline & Universidad Nacional de Colombia Sede Palmira & Palmira \\
\hline
\end{tabular}

Tabla 1. Universidades incluidas en el estudio.

El listado de la Tabla 1 no se puede considerar como un ranking jerarquizado, ya que Sapiens Research (2017a), metodológicamente aborda todos los programas de las universidades en cuestión y no desagrega por facultades ni programas específicos. Aún así, se puede observar la posición que cada universidad ocupa a nivel nacional. En el mismo sentido, el ranking Sapiens Research (2017b), 
no jerarquiza los programas, solo les asigna una calificación como se describió en la metodología.

La información relativa a los grupos de investigación, se presenta en la Tabla 2. En ella se ilustra un consolidado de los grupos de investigación categorizados por Colciencias por cada universi- dad. Adicionalmente, se presenta el resultado de la multiplicación de cada categoría por la ponderación dada y una sumatoria consolidada por universidad. Esta tabla se encuentra organizada en un orden jerárquico de mayor a menor. La universidad con un número mayor es la que presenta mejor indicador de grupos de investigación según la metodología empleada.

\begin{tabular}{|c|c|c|c|c|c|c|c|c|c|c|c|c|c|}
\hline \multirow[b]{2}{*}{ No } & \multirow[b]{2}{*}{ Universidad } & \multicolumn{10}{|c|}{ Clasificación Colciencias } & \multirow{2}{*}{$\begin{array}{l}\text { Total } \\
\text { por } \\
\text { uni- } \\
\text { versi- } \\
\text { dad }\end{array}$} & \multirow[b]{2}{*}{$\begin{array}{l}\text { Consoli- } \\
\text { dado }\end{array}$} \\
\hline & & $\mathbf{A 1}$ & 9,0 & A & 7 & B & 4 & C & 2 & D & 0,1 & & \\
\hline 1 & $\begin{array}{l}\text { Universidad Nacional de Colombia Sede } \\
\text { Bogotá }\end{array}$ & 1 & 9 & 6 & 42 & 3 & 12 & 2 & 4 & 1 & 0,10 & 13 & 67,1 \\
\hline 2 & Universidad de los Andes & 5 & 45 & 0 & 0 & 0 & 0 & 0 & 0 & 0 & 0,00 & 5 & 45 \\
\hline 3 & Universidad del Valle & 1 & 9 & 2 & 14 & 2 & 8 & 4 & 8 & 1 & 0,10 & 10 & 39,1 \\
\hline 4 & Universidad del Norte & 3 & 27 & 1 & 7 & 0 & 0 & 0 & 0 & 0 & 0,00 & 4 & 34 \\
\hline 5 & Universidad de Cartagena & 1 & 9 & 1 & 7 & 3 & 12 & 2 & 4 & 3 & 0,30 & 10 & 32,3 \\
\hline 6 & Universidad EAN & 1 & 9 & 2 & 14 & 0 & 0 & 0 & 0 & 1 & 0,10 & 4 & 23,1 \\
\hline 7 & Universidad de Medellín & 1 & 9 & 0 & 0 & 2 & 8 & 2 & 4 & 1 & 0,10 & 6 & 21,1 \\
\hline 8 & Universidad de la Salle & 0 & 0 & 0 & 0 & 2 & 8 & 3 & 6 & 2 & 0,20 & 7 & 14,2 \\
\hline 9 & Universidad Externado de Colombia & 1 & 9 & 0 & 0 & 0 & 0 & 2 & 4 & 0 & 0,00 & 3 & 13 \\
\hline 10 & Universidad Pontificia Bolivariana & 0 & 0 & 1 & 7 & 0 & 0 & 2 & 4 & 0 & 0,00 & 3 & 11 \\
\hline 11 & Universidad del Cauca & 0 & 0 & 0 & 0 & 2 & 8 & 1 & 2 & 2 & 0,20 & 5 & 10,2 \\
\hline 12 & Universidad de Antioquia & 0 & 0 & 0 & 0 & 2 & 8 & 1 & 2 & 0 & 0,00 & 3 & 10 \\
\hline 13 & Universidad del Rosario & 1 & 9 & 0 & 0 & 0 & 0 & 0 & 0 & 0 & 0,00 & 1 & 9 \\
\hline 14 & Universidad Javeriana & 0 & 0 & 0 & 0 & 2 & 8 & 0 & 0 & 0 & 0,00 & 2 & 8 \\
\hline 15 & Universidad de la Sabana & 0 & 0 & 0 & 0 & 1 & 4 & 1 & 2 & 0 & 0,00 & 2 & 6 \\
\hline 16 & Universidad Militar Nueva Granada & 0 & 0 & 0 & 0 & 1 & 4 & 1 & 2 & 0 & 0,00 & 2 & 6 \\
\hline 17 & $\begin{array}{l}\text { Universidad Pedagógica y Tecnológica } \\
\text { de Colombia }\end{array}$ & 0 & 0 & 0 & 0 & 0 & 0 & 2 & 4 & 1 & 0,10 & 3 & 4,1 \\
\hline 18 & Universidad Santo Tomás & 0 & 0 & 0 & 0 & 0 & 0 & 1 & 2 & 1 & 0,10 & 2 & 2,1 \\
\hline
\end{tabular}

Tabla 2. Clasificación de las universidades según sus grupos de investigación.

El primer resultado a destacar es el de la Universidad de Antioquia. En el ranking nacional de Sapiens Research esta universidad ocupa el segundo lugar, en contraste con el puesto doce según sus grupos de investigación en Administración. Esto indica que los datos de investigación que sustentan a dicha universidad en el primer listado po- drían no reflejar lo referente a los programas de Administración. De igual forma se destaca la sede Bogotá de la Universidad Nacional de Colombia que ocupa el primer lugar en el ranking nacional y en el ranking según sus grupos de investigación en Administración, con lo que mantiene una con- 
gruencia favorable respecto al aporte del indicador investigativo.

En el caso de la Universidad del Valle, destaca un resultado respecto a lo publicado por Hernández-Madroñero et al. (2018). En ese trabajo la mencionada universidad ocupa el primer lugar en cantidad de grupos de investigación, aun cuando es también la universidad que posee más grupos sin reconocimiento por Colciencias (2018, p. 188). La Sede Palmira de la UNAL, es la que posee el peor indicador según grupos de investigación en Administración; esta universidad solo cuenta con un grupo de investigación clasificado al momento de la toma de la información.

Así las cosas, de acuerdo al referente conceptual, por el momento, la Universidad Nacional de Colombia Sede Bogotá, la Universidad de los Andes y la Universidad del Valle son las tres instituciones con mayor plausibilidad, en lo que corresponde al vínculo entre la calidad de la formación y la producción intelectual de las escuelas. Por su parte, la Universidad Pedagógica y Tecnológica de Colombia, la Universidad Santo Tomás y la Universidad Nacional de Colombia Sede Palmira serían las tres universidades con menos posibilidad de ello.

En estos planteamientos $y$, de acuerdo a la revisión bibliográfica, existe un supuesto según el cual a mayor y mejor investigación en los programas, mayor categorización y, por tanto, mejor posición en las clasificaciones. También existe otro supuesto en donde, si se desea una mayor y mejor investigación, es necesario mejorar los procesos de formación investigativa, incluso en el pregrado, en tanto que ello garantice lo anterior (Pineda-Henao, 2018b). Por lo tanto, de acuerdo a los resultados expuestos, las universidades deben mejorar sus procesos de incentivación, control y regulación de la producción de sus grupos de investigación. Esto es aún más importante para las universidades de la parte baja del ranking, en cuanto a sus procesos de mejora.

En el caso de los posgrados hubo datos interesantes. En la Tabla 3 se observan ciertas particularidades. En el primer lugar aparece la Universidad EAN. Esta universidad cuenta con dos doctorados y el mayor número de maestrías afines a la administración del país. La Universidad de los Andes ocupa el tercer lugar con los mismos indicadores que la Universidad Externado de Colombia.

El caso de la UNAL Sede Bogotá es de comentar, pues esta sede ocupa el antepenúltimo lugar en este ranking. En este caso, la facultad a la que pertenece el programa solo cuenta con una maestría en Administración, misma situación de la Sede Palmira. Esto las ubica entre los últimos lugares de la clasificación. Cabe mencionar que al momento de la escritura de este texto, la Universidad Nacional de Colombia se encuentra en proceso de inscripciones para el Doctorado en Administración, en su sede Manizales. 


\begin{tabular}{|c|c|c|c|c|c|c|c|}
\hline \multirow[t]{3}{*}{ zo } & \multirow{3}{*}{ Universidad } & \multicolumn{3}{|c|}{$\begin{array}{l}\text { Total programa } \\
\text { posgrado }\end{array}$} & \multirow{2}{*}{ 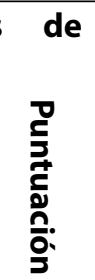 } & \multirow{3}{*}{ 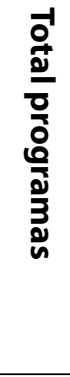 } & \multirow{3}{*}{ 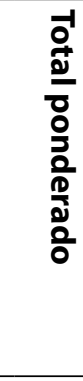 } \\
\hline & & 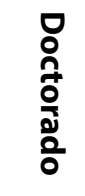 & 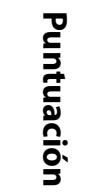 & $\begin{array}{l}3 \\
\mathbf{0} \\
\mathbf{D} \\
\mathbf{2} \\
\mathbf{0}\end{array}$ & & & \\
\hline & & \multicolumn{3}{|c|}{10} & 5 & & \\
\hline 1 & Universidad EAN & 2 & 20 & 9 & 45 & 11 & 65 \\
\hline 2 & Universidad Externado de Colombia & 1 & 10 & 7 & 35 & 8 & 45 \\
\hline 3 & Universidad de los Andes & 1 & 10 & 7 & 35 & 8 & 45 \\
\hline 4 & Universidad Pontificia Bolivariana & 0 & 0 & 6 & 30 & 6 & 30 \\
\hline 5 & Universidad del Norte & 1 & 10 & 4 & 20 & 5 & 30 \\
\hline 6 & Universidad de Medellín & 1 & 10 & 3 & 15 & 4 & 25 \\
\hline 7 & Universidad de la Sabana & 0 & 0 & 4 & 20 & 4 & 20 \\
\hline \multirow[t]{2}{*}{8} & Universidad del Valle & 1 & 10 & 3 & 15 & 4 & 25 \\
\hline & \multicolumn{2}{|l|}{ Universidad Pedagógica y Tecnológica de } & & & & & \\
\hline 9 & Colombia & 0 & 0 & 2 & 10 & 2 & 10 \\
\hline 10 & Universidad Javeriana & 0 & 0 & 3 & 15 & 3 & 15 \\
\hline 11 & Universidad del Rosario & 0 & 0 & 2 & 10 & 2 & 10 \\
\hline 12 & Universidad Santo Tomás & 0 & 0 & 2 & 10 & 2 & 10 \\
\hline 13 & Universidad Militar Nueva Granada & 0 & 0 & 1 & 5 & 1 & 5 \\
\hline 14 & Universidad del Cauca & 0 & 0 & 1 & 5 & 1 & 5 \\
\hline 15 & Universidad de Antioquia & 0 & 0 & 1 & 5 & 1 & 5 \\
\hline \multirow[t]{2}{*}{16} & Universidad de la Salle & 0 & 0 & 1 & 5 & 1 & 5 \\
\hline & \multicolumn{2}{|l|}{ Universidad Nacional de Colombia Sede } & & & & & \\
\hline 17 & Bogotá & 0 & 0 & 1 & 5 & 1 & 5 \\
\hline \multirow[t]{2}{*}{18} & Universidad de Cartagena & 0 & 0 & 1 & 5 & 1 & 5 \\
\hline & Universidad Nacional de Colombia Sede & & & & & & \\
\hline 19 & Palmira & 0 & 0 & 1 & 5 & 1 & 5 \\
\hline
\end{tabular}

Tabla 3. Clasificación de las universidades según sus programas de posgrado.

Si se considera por separado el criterio de los posgrados y se relaciona la cantidad y calidad de estos con la formación investigativa de excelencia de la planta docente, según los referentes conceptuales aquí expuestos, se puede interpretar que la Universidad EAN, La Universidad Externado de Colombia y la Universidad de los Andes presentan mejor influencia en la formación de calidad en los programas de pregrado. Por su parte, las Sedes Bogotá y Palmira de la Universidad Nacional de
Colombia y la Universidad de Cartagena serían las peor clasificadas bajo dicho criterio.

En la etapa de búsqueda de información, en el criterio de programas de posgrado fue notorio un alto número de especializaciones por parte de las facultades de Administración (sobre todo en universidades privadas), en consonancia con Salcedo Serna et al (2017). Sin embargo, estos programas de especialización no se tuvieron en cuenta porque, dada su naturaleza, ellos no promueven la 
generación de nuevo conocimiento. En este sentido, las facultades que deseen un mayor desarrollo del conocimiento y mejores clasificaciones en esta área, deberían preocuparse por la apertura de más programas de maestría y doctorado con alta calidad.

Una vez hallados los resultados de los dos criterios, se procedió a la creación del ranking final, ver Tabla 4. La Universidad de los Andes lidera la clasificación con un ponderado final de 45 puntos. Sus indicadores de posgrado y grupos de investigación son semejantes. Aun cuando la Universidad Nacional de Colombia Sede Bogotá presenta el mejor indicador respecto a grupos, su indicador frente a los posgrados la relega a un tercer lugar en la clasificación, donde es superada por la Universidad EAN.

\begin{tabular}{|c|c|c|c|c|c|c|}
\hline No & Universidad & $\begin{array}{l}\text { Puntaje } \\
\text { Posgrados }\end{array}$ & $\begin{array}{l}\text { Ponderación } \\
50 \%\end{array}$ & $\begin{array}{l}\text { Puntaje } \\
\text { Grupos de } \\
\text { investigación }\end{array}$ & $\begin{array}{l}\text { Ponderación } \\
50 \%\end{array}$ & $\begin{array}{l}\text { Total } \\
\text { ponderado }\end{array}$ \\
\hline 1 & Universidad de los Andes & 45 & 22,5 & 45 & 22,5 & 45 \\
\hline 2 & Universidad EAN & 65 & 32,5 & 23,1 & 11,55 & 44,05 \\
\hline 3 & $\begin{array}{l}\text { Universidad Nacional de Colombia } \\
\text { Sede Bogotá }\end{array}$ & 5 & 2,5 & 67,1 & 33,55 & 36,05 \\
\hline 4 & Universidad del Valle & 25 & 12,5 & 39,1 & 19,55 & 32,05 \\
\hline 5 & Universidad del Norte & 30 & 15 & 34 & 17 & 32 \\
\hline 6 & $\begin{array}{l}\text { Universidad Externado de Colom- } \\
\text { bia }\end{array}$ & 45 & 22,5 & 13 & 6,5 & 29 \\
\hline 7 & Universidad de Medellín & 25 & 12,5 & 21,1 & 10,55 & 23,05 \\
\hline 8 & Universidad Pontificia Bolivariana & 30 & 15 & 11 & 5,5 & 20,5 \\
\hline 9 & Universidad de Cartagena & 5 & 2,5 & 32,3 & 16,15 & 18,65 \\
\hline 10 & Universidad de la Sabana & 20 & 10 & 6 & 3 & 13 \\
\hline 11 & Universidad Javeriana & 15 & 7,5 & 8 & 4 & 11,5 \\
\hline 12 & Universidad de la Salle & 5 & 2,5 & 14,2 & 7,1 & 9,6 \\
\hline 13 & Universidad del Rosario & 10 & 5 & 9 & 4,5 & 9,5 \\
\hline 14 & Universidad del Cauca & 5 & 2,5 & 10,2 & 5,1 & 7,6 \\
\hline 15 & Universidad de Antioquia & 5 & 2,5 & 10 & 5 & 7,5 \\
\hline 16 & $\begin{array}{l}\text { Universidad Pedagógica y Tecnoló- } \\
\text { gica de Colombia }\end{array}$ & 10 & 5 & 4,1 & 2,05 & 7,05 \\
\hline 17 & Universidad Santo Tomás & 10 & 5 & 2,1 & 1,05 & 6,05 \\
\hline 18 & Universidad Militar Nueva Granada & 5 & 2,5 & 6 & 3 & 5,5 \\
\hline 19 & $\begin{array}{l}\text { Universidad Nacional de Colombia } \\
\text { Sede Palmira }\end{array}$ & 5 & 2,5 & 0,1 & 0,05 & 2,55 \\
\hline
\end{tabular}

Tabla 4. Clasificación de las mejores universidades colombianas en Administración.

De acuerdo con estos resultados, y al unificar los criterios de los referentes conceptuales, la Universidad de los Andes, la Universidad EAN y la Universidad Nacional de Colombia Sede Bogotá, serían las universidades con más favorabilidad de una influencia positiva de la investigación en la formación de calidad en el pregrado.

Al comparar los indicadores de esta propuesta con la clasificación de Sapiens Research, se detectan 
ciertas variaciones. Las más evidentes son las de la Universidad EAN, la Universidad de Antioquia y la Universidad Pedagógica y Tecnológica de Colombia. En el caso de la primera, pasó de estar en el puesto 32 a segunda del ranking. Caso contrario el de las otras dos universidades, que en el ranking general hacen parte del top 10 , mientras que para los indicadores en administración aparecen en los puestos 15 y 16 , respectivamente.

La universidad Nacional de Colombia Sede Palmira mantiene en ambos casos la última posición entre las diecinueve universidades estudiadas. Esta situación podría explicarse dado el tamaño de la Sede y del programa de Administración, respecto a las demás universidades. También puede deberse al hecho posible de que los grupos de investigación no se hayan presentado a las convocatorias de Colciencias de donde se obtuvieron los datos.

Lo anterior significa que, si las facultades de Administración con peores calificaciones desean mejorar su posición en el ranking, deben tener una mejor proporción entre los dos criterios, i.e. entre la cantidad y calidad de grupos de investigación y cantidad de posgrados. En razón a ello, la Universidad Nacional de Colombia, sedes Bogotá y Palmira, podría mejorar sus posiciones con la apertura de más programas de posgrado y la mejora de su gestión en los grupos de investigación; esto último, sobre todo, en el caso de la Sede Palmira.

Los resultados anteriores sugieren que, dadas las diferencias manifiestas entre estos datos y lo expuesto en los rankings, se debe tener una mayor cautela a la hora de analizar las diferentes clasificaciones de las universidades y la calidad de los programas en lo que respecta a la investigación. Esto en la medida que algunas de ellas pueden mezclar muchas variables mientras que otras pueden estar omitiéndolas, con lo que se pierde parcialidad.

Tanto estos resultados como la metodología acá empleada, son de utilidad para mejorar los procesos de estructuración curricular de los programas y de evaluación de los mismos, por parte de enti- dades como el Consejo Nacional de Acreditación, CNA. Esta entidad podría, por ejemplo, usarlos y mejorarlos a partir de la información con la que dispone, con el fin de medir mejor la calidad y proponer mejoras para los programas de Administración, e incluso de otras disciplinas.

La utilidad de la metodología presentada radica en que propone una manera de medir y ponderar parámetros de calidad en los programas a partir de información objetiva. Sin embargo, la metodología está sujeta a mejoras a partir de la inclusión de más variables, susceptibles de ser cuantificadas, como: los resultados de las Pruebas Saber PRO del ICFES, las políticas institucionales y datos sobre empleabilidad y desempeño profesional de los egresados. También es necesario desarrollar teorías o estudios, que permitan valorar la mayor o menor importancia de los criterios evaluados entre sí, con el fin de dar paso a ponderaciones más justas y precisas. Con todo lo anterior, la clasificación acá expuesta podría sufrir algunos cambios.

\section{Conclusiones}

El propósito de la presente investigación fue aproximarse a un panorama del estado de algunos de los mejores programas de pregrado en Administración, de acuerdo a indicadores de investigación, relativos a los criterios de: a) calidad y cantidad de grupos de investigación, y b) programas de posgrado. Se obtuvo un listado de diecinueve universidades en el que, de acuerdo a los referentes conceptuales, las primeras representarían las de mejor influencia de la investigación en la formación de calidad de sus programas de pregrado en Administración.

Es evidente que aun cuando las universidades estudiadas son las mejores en indicadores de calidad en investigación, parece ser que los programas de Administración de algunas de ellas, como en el caso de la Universidad de Antioquia, no parecen aportar a las cifras globales, de acuerdo a los datos recopilados y los criterios establecidos en el estudio. 
Igualmente, se sugiere, para futuras investigaciones, examinar los enfoques de cada programa de pregrado en Administración, ya que se podría suponer que no todos sus currículos están enfocados hacia el fomento de la investigación, como lo plantearon Pineda-Henao (2018b) y Giraldo-López (2018).

Asimismo, los criterios usados podrían omitir otros ítems que no se hayan contemplado en el referente conceptual. Además, se debe verificar los resultados académicos de estos programas curriculares respecto a los pregrados de Administración con mejores índices en este ranking. Para ello habría que analizar si los métodos como el Saber PRO son fidedignos y demuestran la situación real de los futuros egresados. Igualmente, sería interesante poder constatar la influencia de la investigación, en la calidad y el desempeño profesional de los egresados. Los aspectos anteriores representan temáticas de investigación para futuros estudios.

Sería pertinente que organizaciones como la Asociación Colombiana de Facultades de Administración, ASCOLFA, o el CNA, realizaran un ranking con la información oficial a la que tienen acceso, específicamente en los programas de pregrado en Administración utilizando esta metodología. Esto serviría de retroalimentación para las mismas facultades a la hora de la planificación de sus programas, con lo que podría mejorarse la calidad y cantidad de la investigación.

En cuanto a los inconvenientes que podrían plantear la clasificación aquí presentada, cabe mencionar la tendencia que tienen las diferentes escuelas o facultades a la transversalidad y a la transdisciplinariedad entre los diferentes grupos y programas. Lo anterior ocasiona una inexactitud a la hora de seleccionar grupos de investigación y programas de posgrado en Administración. El segundo inconveniente es la desactualización que podría presentar la información disponible en las páginas web de algunas universidades o el celo con el que podrían compartirla. Esto también podría ocasionar inexactitudes que perjudicarían el resultado de los estudios.

\section{Agradecimientos}

Al Grupo de Estudios Neoinstitucionales - GEN, en especial a los estudiantes: Isabella Hernández Madroñero, Luisa Rengifo Pasiminio, Libardo Acosta y Mateo Ortega, por su colaboración en la captura y análisis de la información. Igualmente, se hace un reconocimiento especial a los profesores Carlos Tello Castrillón y Marco Alexis Salcedo, que lideraron los proyectos de investigación. Finalmente, se resaltan los aportes dados en el proceso de evaluación y arbitraje para la mejora de este documento.

\section{Referencias}

Aldana-de Becerra, G. M. (2012). La formación investigativa: su pertinencia en pregrado. Revista Virtual Universidad Católica del Norte, 1 (35), 367379. Recuperado de: http://revistavirtual.ucn.edu. co/index.php/RevistaUCN/article/view/366/681

Calderón-Hernández, G., Castaño-Duque, G. A., Lozada-Barahona, N. E., Gutiérrez-Vargas, L. M., Pérez-Herrera, P. A., \& Posada-Bernal, R. (2014). Generación de conocimiento en los grupos élite de investigación en administración en Colombia. Bogotá, Colombia: UNAL - ASCOLFA.

Calderón-Hernández, G., Gutiérrez-Vargas, L. M., \& Castaño-Duque, G. A. (2017). La investigación en las facultades de administración de Colombia. Revista Lasallista de Investigación, 14 (1), 42-55. doi: https://doi.org/10.22507/rli.v14n1a3

Carvajal, J. G. (2010). El concepto de organización para la administración. Ontología y epistemología de las organizaciones. En G. Calderón Hernández \& G. A. Castaño Duque, Investigación en administración: conocimiento para el bienestar de las personas y el desarrollo de las organizaciones, 15-21. Manizales, Colombia: Editorial UNAL. 
Colciencias. (2015, enero). Convocatoria Nacional para el Reconocimiento y Medición de Grupos de Investigación, Desarrollo Tecnológico o de Innovación y para el Reconocimiento de Investigadores del SNCTel, 2015. Recuperado de: http://www.colciencias.gov.co/sites/default/files/upload/paginas/ conv-medicion-737-2015.pdf

Cornella-Solans, A. (1998). ¿Es el management una ciencia? un programa de diez preguntas para reflexiona. Revista EAN, (35), 4-20. Recuperado de: https://journal.universidadean.edu.co/index.php/ Revista/article/view/864

Cruz Kronfly, F. (1982). Hacia una redefinición del concepto de organización. En H. Galvis Parrasi, De lo humano organizacional. Facultad de Ciencias de la Administración, Universidad del Valle.

Dávila, C. (1982). Lineamientos y experiencias sobre una política de investigación en las escuelas de administración de America Latina 77-87. I/ Encuentro de investigadores en administración. Cali, Colombia: Universidad del Valle.

Dávila, C. (1986). La investigación en Administración: anotaciones sobre la experiencia colombiana y políticas para su promoción a nivel nacional y latinoamericano. Revista Cátedra, 1, 13-27.

Dávila, C. (1988). La investigación en administración: Barreras y perspectivas. Academia, (19).

Durango-Yepes, C. M. (2008). Mitos sobre la naturaleza de la investigación en administración en Colombia y otros países latinoamericanos. Revista ciencias estratégicas, 16 (19), 23-32. Recuperado de: https://revistas.upb.edu.co/index.php/cienciasestrategicas/article/view/592/530

Flórez, D. T. (2011). Epistemología y Administración. En J. G. Carvajal., \& J. A. Izasa, Memorias Encuentro de la red en filosofía, teoría y educación en administración Manizales, mayo 11 - 13 de 2011. 4863. Manizales, Colombia: UNAL - Manizales.
Gantman, E. R. (2013). Escuelas de negocios latinoamericanas Un análisis empírico. Contaduría y Administración, 58 (3), 279-298. Recuperado de: https://www.sciencedirect.com/science/article/pii/ S0186104213712300

Giraldo-López, A. R. (2018). La influencia de la malla curricular en la formación investigativa en programas de administración en Colombia (Tesis pregrado). Universidad Nacional de Colombia sede Palmira, Palmira, Valle del Cauca.

Hernández-Madroñero, I., Londoño-Cardozo, J., \& Pineda-Henao, E. F. (2018). Clasificación de grupos de investigación en administración de los mejores programas de administración de Colombia. Revista Ensayos, 10, 181-193. Recuperado de: https:// revistas.unal.edu.co/index.php/ensayos/article/ view/72505/66255

Hernández-Martínez, A., Saavedra-Mayorga, J. J., \& Sanabria-Rangel, M. (2006). La formación administrativa en Colombia: el caso de las maestrías. Revista Facultad de Ciencias Económicas, XIV (2), 21-38. Recuperado de: http://www.redalyc.org/articulo. oa?id=90900204

Londoño-Cardozo, J. D. (2018). Educación para emprender ¿práctica o teoría?. Revista Argentina de Investigación en Negocios, 4 (1), 97-106. Recuperado de: http://ppct.caicyt.gov.ar/index.php/rain/ article/view/V4n1a8/pdf

Malaver-Rodríguez, F. (1999). La investigación en gestión empresarial. Academia. Revista Latinoamericana de Administración, (23), 62-77. Recuperado de: http://www.redalyc.org/articulo. oa?id=71602306

Malaver-Rodríguez, F. (2006). El despegue de la investigación Colombiana en Administración: Análisis de sus avances en el período 2000-2006. Cuadernos de Administración 19 (32) 71-109. Recuperado de: http://www.redalyc.org/pdf/205/20503204. pdf 
Malaver-Rodríguez, F., \& López-Gallego, F. (2016). La investigación sobre la administración en Colombia (1965-2015): balance y perspectivas. Cuadernos de Administración, 29 (52), 141-166. doi: https://doi.org/10.11144/Javeriana.cao29-52.iacb

Marín-Idárraga, D. A. (2012). Consideraciones epistemológicas en torno al carácter científico de la administración. Innovar, 22(46), 39-52. Recuperado de: https://revistas.unal.edu.co/index.php/innovar/article/view/39323/41226

Ministerio de Educación Nacional de Colombia. Decreto 1295, Pub. L. No. 1295, 17 (2010). Recuperado de: https://genunpal.page.link/ZD5a

Ogliastri, E. (1982). Investigaciones en Administración 121-127. II Encuentro de investigadores en administración. Cali, Colombia: Universidad del Valle.

Pineda-Henao, E. F. (2012). Filosofía de la ciencia aplicada a la administración. Revista Ensayos, 5 (5), 177-192. Recuperado de: https://revistas.unal.edu. co/index.php/ensayos/article/view/51044

Pineda-Henao, E. F. (2013). La praxis administrativa y el orden. Una noción de la práctica administrativa como ordenación instrumental, sistemática e intencional de las organizaciones. Ensayos. Revista de los Estudiantes de Administración de Empresas, 6 (1), 11-28. Recuperado de: https://revistas.unal. edu.co/index.php/ensayos/article/view/49853

Pineda-Henao, E. F. (2014a). Ontología de la administración: Una aproximación a la práctica administrativa como hecho institucional. Ensayos. Revista de los Estudiantes de Administración de Empresas, 7 (1), 122-143. Recuperado de: https://revistas.unal. edu.co/index.php/ensayos/article/view/50448

Pineda-Henao, E. F. (2014b). Una fundamentación ontológica de la práctica administrativa como técnica social ordenadora institucionalizada (Tesis pregrado). Universidad Nacional de Colombia, Palmi- ra, Valle del Cauca. Recuperado de https://goo.gl/ TfCqJp

Pineda-Henao, E. F. (2015). Algunas distinciones conceptuales entre ciencia y tecnología para el problema del estatus de la administración. En J. G. Carvajal, Encuentro de la Red en Filosofía, Teoría y Educación en Administración, 10-19. Manizales, Colombia: UNAL.

Pineda-Henao, E. F. (2017). Disciplina administrativa y práctica administrativa: una perspectiva analítica del problema del estatus epistemológico de la administración (Tesis pregrado). Universidad del Valle, Cali.

Pineda-Henao, E. F. (2018a). Administración y organizaciones: una mirada más allá de las fronteras de lo instrumental. En C. Tello-Castrillón., \& E. F. Pineda-Henao, Conjeturas organizacionales: fundamentos para el estudio de la organización (Primera Edición, pp. 31-54). Bogotá: Editorial UNAL.

Pineda-Henao, E. F. (2018b). Sobre la formación investigativa: diagnóstico comparativo del programa de Administración de la Universidad Nacional de Colombia Sede Palmira (Tesis Maestría). Universidad Nacional de Colombia, Palmira, Valle del Cauca.

Pineda-Henao, E. F., \& Tello-Castrillón, C. (2018). ¿Ciencia, técnica y arte?: análisis crítico sobre algunas posturas del problema del estatus epistemológico de la Administración. Revista Logos Ciencia \& Tecnología, 10 (4), 112-130.

Pontificia Universidad Javeriana (2017a). Administración de Empresas. Recuperado de: https://goo. $\mathrm{gl} / \mathrm{szDk} 8 \mathrm{c}$

Pontificia Universidad Javeriana (2017b). Grupos de Investigación - Facultad de Ciencias Económicas y Administrativas (Institucional). Recuperado de: http://cea.javeriana.edu.co/investigacion-publicaciones/grupos-investigacion 
Salcedo-Serna, M. A., Londoño-Cardozo, J. D., \& Hernández-Madroñero, I. (2017). Tendencias de formación posgradual y áreas de investigación en Administración de Empresas. Estudio comparativo en América. Revista Logos Ciencia \& Tecnología, 9 (2), 134-150. doi: https://doi.org/10.22335/rlct. v9i2.442

Sapiens Research (2017a, mayo). Ranking U-Sapiens 2016-2. Recuperado de: http://www.sapiensresearch.org/usapiens/2016-2

Sapiens Research (2017b, junio). Ranking Pre-Sapiens 2016-1. Recuperado de: http://www.sapiensresearch.org/presapiens/2016-1

Universidad de Antioquia (2017a). Estos son todos los programas de pregrado de la UdeA. Recuperado de: https://goo.gl/mULinD

Universidad de Antioquia (2017b). Investigación (Institucional). Recuperado de: https://goo.gl/ wqN77g

Universidad de Cartagena (2017a). Facultad de Ciencias Económicas - Administración de Empresas. Recuperado de: https://goo.gl/4Ydajr

Universidad de Cartagena (2017b). Investigaciones Universidad de Cartagena - Ciencias Económicas (Institucional). Recuperado de: http:// investigaciones.unicartagena.edu.co/index.php/ grupos-de-facultades/ciencias-economicas

Universidad de la Sabana (2017). Estudiar carrera de Administración de Empresas. Recuperado de: https://goo.gl/JPMYXM

Universidad de la Salle (2017a). Administración De Empresas. Recuperado de: https://goo.gl/Ty1M5K

Universidad de la Salle (2017b). Grupos de Investigación. Recuperado de: https://www.lasalle.edu. co/investigacion/grupos-de-investigacion
Universidad de Los Andes (2015a). Doctorado en Administración - Facultad de Administración. Recuperado de: https://goo.gl/40SOmB

Universidad de Los Andes (2015b). Especializaciones - Facultad de Administración. Recuperado de: https://goo.gl/YeYLU2

Universidad de Los Andes (2015c). MBA - Facultad de Administración. Recuperado de: https://goo.gl/ MbRVgJ

Universidad de Los Andes (2017). Información General - Facultad de Administración. Recuperado de: https://goo.gl/tZDtXg

Universidad de Medellín (2017a). Administración de empresas - Información general. Recuperado de: https://goo.gl/DPjWAu

Universidad de Medellín (2017b). Investigación (Institucional). Recuperado de: https://www. udem.edu.co/index.php/investigacion

Universidad del Cauca (2017a). Administración de Empresas. Recuperado de: https://goo.gl/cpd9p2

Universidad del Cauca (2017b). Vicerrectoría de Investigaciones - Grupos de Investigación. Recuperado de: http://vri.unicauca.edu.co/index.php/ gruposinvestigacion

Universidad del Norte (2015). Catálogo de Programas - Universidad del Norte. Recuperado de: https://goo.gl/1oiOxn

Universidad del Norte (2017a). Carrera, Programa de Administración de Empresas Barranquilla. Recuperado de: https://goo.gl/sg3U8K

Universidad del Norte (2017b). Grupos de Investigación - Escuela de Negocios (Institucional). Recuperado de: https://www.uninorte.edu.co/web/ escuela-de-negocios/grupos-de-investigacion 
Universidad del Rosario (2017a). Plan de estudios Administración de Empresas. Recuperado de: https://goo.gl/cceUSh

Universidad del Rosario (2017b). Publicaciones e Investigaciones en Administración [Institucional]. Recuperado de: http://www.urosario.edu.co/Escuela-de-Administracion/Investigacion/Publicaciones/

Universidad del Valle (2013). Administración de Empresas - Facultad de Ciencias de la Administración. Recuperado de: https://goo.gl/i3nuyz

Universidad del Valle (2015). Facultad de Ciencias de la Administración. Recuperado de: https://goo. $\mathrm{gl} / \mathrm{s} 9 \mathrm{ilcj}$

Universidad EAN (2017a). Gerencia de Investigaciones (Institucional). Recuperado de: https://universidadean.edu.co/es/investigacion

Universidad EAN (2017b). Pregrado en Administración de Empresas - Presencial, Ofertas académicas. Recuperado de: https://goo.gl/9UJYvm

Universidad Externado de Colombia (2015). Conoce nuestros distintos programas de posgrado. Recuperado de: https://goo.gl/cklaPg

Universidad Externado de Colombia (2017a). Investigación (Institucional). Recuperado de: http:// investigacion.uexternado.edu.co/grupos/

Universidad Externado de Colombia (2017b). Pregrado en Administración de Empresas. Recuperado de: https://goo.gl/ueoLYo

Universidad Militar Nueva Granada (2017). Administración de Empresas - UMNG. Recuperado de: https://goo.gl/1DpkHx

Universidad Nacional de Colombia (2015). Repositorios Pregrado y Posgrado. Recuperado de: https://goo.gl/e6ExrU
Universidad Nacional de Colombia, Sede Bogotá (2009). Plan de Estudios - Administración de empresas FCE. Recuperado de: https://goo.gl/j4ov8A

Universidad Nacional de Colombia, Sede Palmira (2017). Facultad de Ingeniería y Administración. Recuperado de: http://www.palmira.unal.edu.co/ index.php/facultades/ingenieria-administracion

Universidad Pedagógica y Tecnológica de Colombia (2017). Grupos de Investigación de la UPTC [Institucional]. Recuperado de: http://www.uptc. edu.co/direccion_investigaciones/cent_grupos/ grup_inv/index.html

Universidad Pedagógica y Tecnológica de Colombia (2018). Administración de Empresas. Recuperado de: https://goo.gl/8S5yzp

Universidad Pontificia Bolivariana (2017a). Administración de Empresas. Recuperado de: https:// goo.gl/2b5A1m

Universidad Pontificia Bolivariana (2017b). Grupos de Investigación de la Universidad Pontificia Bolivariana (Institucional). Recuperado de: https:// www.upb.edu.co/es/investigacion/grupos-de-investigacion

Universidad Santo Tomás (2017a). Grupos de investigación (Institucional). Recuperado de: http:// unidadinvestigacion.usta.edu.co/index.php/procesos-de-gestion/grupos-de-investigacion

Universidad Santo Tomás (2017b). Pregrado en Administración de Empresas. Recuperado de: https://goo.gl/yn8Ugm 